\title{
Non Linear Distortions introduced by Amplifiers on COFDM signals. Measurements, Effects and Compensation Techniques
}

\author{
S Andreoli ${ }^{1}$, P Banelli ${ }^{2}$, A Longaroni ${ }^{1}$ and C Massini ${ }^{1}$ \\ 1 Itelco R\&D, Orvieto (TR), Italy e.mail:itelcord@krenet.it \\ 2 Istituto di Elettronica, Università di Perugia, Italy \\ e-mail: banelli@istel.ing.unipg.it
}

\begin{abstract}
Coded Orthogonal Frequency Division Multiplexed (COFDM) techniques, used by terrestrial European broadcasting systems (DAB, DVB-T), could be adopted in the last mile of the downlink channel for Wireless Broadband Communication System to provide Multimedia Services to mobile or nomadic users by cellular coverage techniques, as evinced from the actual research activity. COFDM signals are characterised by non-constant envelope (peak-to-mean power ratio > $10 \mathrm{~dB}$ ) which forces to project amplifiers with low or well-counteracted nonlinear distortion effects. Our aim is to present Itelco experience to characterise non-linear distortions introduced by final amplifiers on COFDM signals (AM/AM, $A M / P M$ and NonLinearities with Memory). Both experimental measurements and simulation results will be shown. Moreover we will point out the improvements achievable by digital baseband predistortion, to counteract for AM/AM and $A M / P M$ non-linearities, and by $R F$ compensation techniques to remove Non Linear Memory effects.
\end{abstract}

\section{Introduction}

The actual trend in Communication Systems is toward the development of broadband network for the distribution of multimedia and interactive services. Wireless Networks represent an attractive technical solution to this task in countries that lack cable infrastructure and where the costs or time to realise a classical network is too high. Moreover, wireless network can easily respond to the increasing demand to extend broadband services to mobile or nomadic users. The intrinsic low channel capacity of wireless systems (compared to the cable network), due to the limits of radio frequency spectrum, can be overcome by the use of cellular coverage techniques extensively employed in mobile telephone systems. Network architecture with a last-mile service distribution obtained by RF cellular topology and with network backbones, alternatively realised by RF pointto-point link or cable infrastructure, could be a possible scenario. The Multipoint Multichannel Distribution Service (MMDS) and the Local Multipoint Distribution 
Service (LMDS) that respectively operate at [2.150-2.168] GHz and [27.5-29.5] $\mathrm{GHz}$, are two possible approaches to that philosophy of service distribution [Ref. 1]. Recent activities in CABSINET [Ref. 2] European research project are focused on the effectiveness of a 2 Layer network architecture. The first layer for fixed users with $40 \mathrm{GHz}$ macrocell coverage and the second layer for mobile or nomadic users in indoor environment with $5 \mathrm{GHz}$ microcell coverage. The system architecture is thought to accomplish for compatibility with Digital Video Broadcasting (DVB) standards: Microwave Satellite (DVB-MS) QPSK modulation is used for the macrocell coverage, while Terrestrial (DVB-T) COFDM is employed for the microcell mobile environment [Ref. 3]. For this reason in this paper, we concentrate our attention on COFDM signals distortions. COFDM signals are characterised by a highly variable envelope and therefore they are very sensitive to non-linear distortions. This fact forces the engineers to project power amplifiers where non-linear distortion phenomena are avoided or well counteracted. In the following we will consider and analyse non-linear distortions introduced by a power amplifier on a single Digital Audio Broadcasting (DAB) channel [Ref. 4], but the same considerations and conclusions can be extended to DVB-T signals. We choose to consider a multichannel microcell transmitter with single channel amplifiers. This configuration has the advantage to allow the compensation of every single amplifiers, by the use of digital baseband predistortion techniques on every DAB (DVB-T) channel. Vice versa, the architecture with a single wideband amplifier, because of the high sample frequency needed, makes impossible a digital predistortion and forces to only use RF analog non-linear compensation techniques.

\section{Non Linear Distortions}

It is possible to classify non-linear distortions as instantaneous (without memory) or with memory. If $\mathrm{x}(\mathrm{t})$ expressed by

$$
x(t)=A(t) \cos \left[\omega_{o} t+\theta(t)\right]
$$

represents the input to a non-linear RF amplifier, the distorted output $y(t)$ is in general expressed by

$$
y(t)=g[x(t)]
$$

where $\mathrm{y}(\mathrm{t})$ depends on the instantaneous input value only. In such situation, when all the circuit time constants are much smaller than the reciprocal value of the maximum envelope frequency, the RF band-pass output of a non-linear amplifier can be expressed by

$$
y_{f}(t)=G[A(t)] \cos \left\{\omega_{o} t+6(t)+\Phi[A(t)]\right\}
$$

where $G$ and $\Phi$, that respectively represent the AM/AM and AM/PM distortion curves, summarise the distortion effects over the fundamental output component. The instantaneous non-linearity $\mathrm{g}(\mathrm{x})$ is related to $\mathrm{G}(\mathrm{A})$ and $\Phi(\mathrm{A})$ by Chebyschev 
Transform [Ref. 5]. The output $y(t)$ of a non-linear device with memory does not only depend on the actual value $\mathrm{x}(\mathrm{t})$, but also on the previous ones. This kind of systems can be generally modelled by Volterra Series Expansions [Ref. 6]. Unfortunately the practical measurement of the Volterra Kernels is a hard task and it represents the limit to the potentiality of this approach. It is possible to overcome this impairment when the memory of the system and the non linearity are completely separated, like in a digital single carrier communication system [Ref. 7 and 8]. However as this is not the situation, we will introduce a different approach to model the non-linear distortions with memory of power amplifiers.

\section{Modelling of Power Amplifiers by Measurements}

In this section two different kind of measurements are presented: the first one outlines the instantaneous non-linear distortions by AM/AM and AM/PM characterisation, the other one points out memory effects that can be combined with non-linear distortions. AM/AM and AM/PM respectively represent how the gain and the phase of the output signal change for different values of the input power. The measurements performed on a Class AB amplifier for III-Band DAB transmission are shown in Fig.1. The measurements were made at different frequencies spanning much more than the $1.5 \mathrm{MHz}$ DAB signal bandwidth, in order to reveal any memory effects introduced by the filtering stages of the amplification chain. We found that AM/AM and AM/PM do not vary significantly over a frequency span wider than the signal band. This result excludes any kind of memory introduced by the filtering stages but it is not enough to assure that some other non linear memory effects could seriously degrade the quality of the COFDM signals. Indeed the high variable envelope of COFDM signals is critical for not only AM/AM and AM/PM distortions, but also for memory distortions.
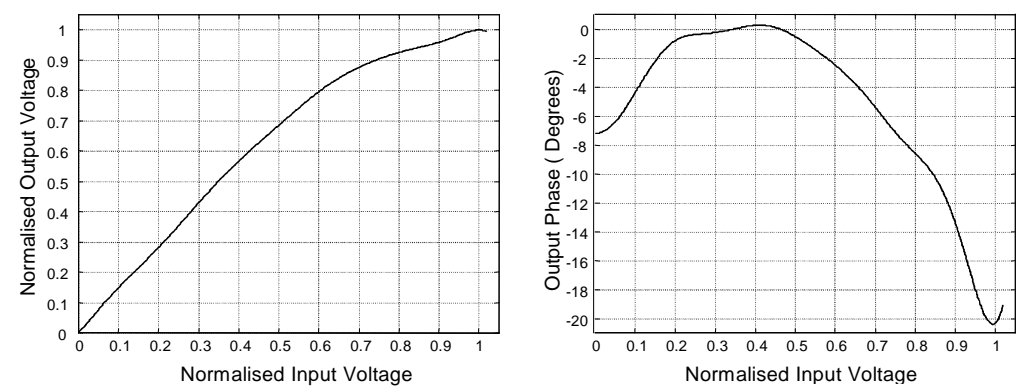

Fig. 1 AM/AM and AM/PM Measured Distortion Curves

This kind of signal causes great problems to the amplifiers because of rapid thermal variations, biasing fluctuations, and power supply requirements. This implies that other non-linear effects could be superimposed and mixed to the classical AM/AM and AM/PM, making the distortions to depend on thermal, biasing or supply conditions. All these circumstances can be generally seen as a 
variation of the amplifier operating point.

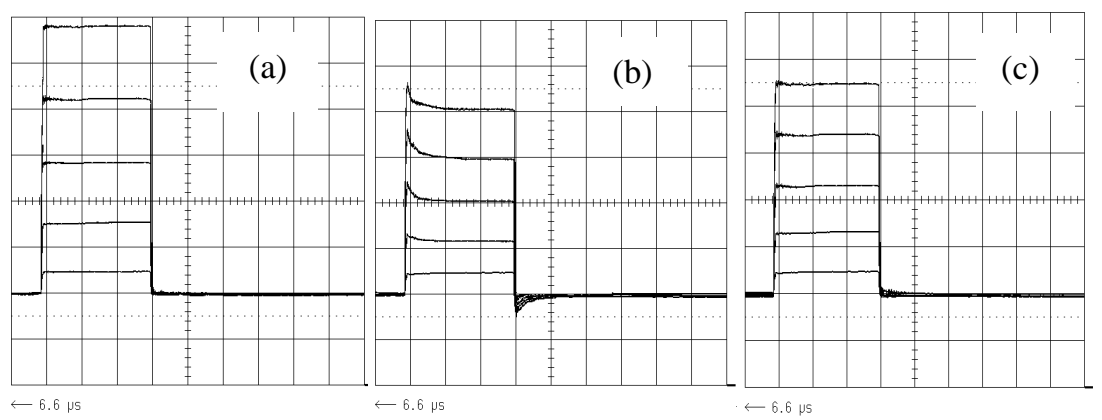

Fig. 2 Envelope Distortion (a) Input (b) Output Compression with Memory (c) Output Compression with RF Memory Compensation

This fact is well outlined by a particular input-output measurement made on the considered Class $\mathrm{AB}$ amplifier and shown in Fig.2. The amplifier exhibits a transient state, with a time dependent input-output gain, after every variation of the signal envelope. The system memory is evident because such a distortion also depends on the previous values of the signal. A distortion phenomenon like the one shown in Fig. 2 occurs every time the variation speed of the input signal (maximum envelope frequency) is faster than the system time response to this variation. The test is used to show how the amplifier responds to these rapid signal variations, even if the test signal itself is not OFDM modulated. Moreover, Fig.2 shows that the memory effect is combined to the AM/AM distortion because of the gain compression that corresponds to the input power growth. The previous considerations suggest that the classical AM/AM and AM/PM characterisation is not enough to describe the input-output relationship for such a kind of amplifiers. It is possible to assume however that the amplifier is represented by a set of $\mathrm{AM} / \mathrm{AM}$ and AM/PM curves. Each couple of curves is associated with different values of the changing parameters or, equivalently, with a different operating point. It means that the AM/AM and AM/PM become 3D surfaces with a double input-domain. An input is the classical instantaneous envelope, the other is the actual operating point parameter that changes depending on the signal history. Because of the tight space available, a more detailed description of the model will be object of a future work.

\section{Non Linear Distortions on OFDM Signals and Counteraction}

Intermodulation between the orthogonal carriers occurs when OFDM signals pass trough a non-linear device generating signal distortion. It is possible to distinguish between In-Band and Out-Band distortions. The first one is responsible for BER performance degradation while the second for Adjacent Channel Interference (ACI). The ratio between intermodulation power and signal power grows with the 
output power, forcing the amplifier to work with an Output Back Off (OBO) from the maximum power. Baseband predistortion is a well-known technique for the compensation of AM/AM and AM/PM distortions introduced by RF power amplifiers [Ref. 9 to 13]. By use of this technique, the magnitude $A(t)$ of the input signal is predistorted by two curves $\mathrm{H}(\mathrm{A})$ and $\Psi(\mathrm{A})$ that globally invert $\mathrm{G}(\mathrm{A})$ and $\Phi(\mathrm{A})$ of (2.3) [Ref. 5]. The cascade of the predistorting device with the amplifier gives rise to a global system without AM/PM and with a residual AM/AM that can be modelled as a soft limiter. Therefore, it is not possible to avoid the clipping [Ref. 14] introduced on the signal envelope even if an ideal predistortion is achieved. In practice OFDM signals also force the predistorted amplifier, to work whit a certain OBO. The predistorter action, however, even if effective in memory-less conditions, may be completely vanished if the amplifier exhibits any non-linear memory distortions, like that shown in Fig.2(b). Indeed the baseband predistorter we have realised is based on 1-dimensional memory store of the AM/AM and AM/PM inversion [Ref. 13]. N-dimensional look-up tables would be needed if memory effects have to be counteracted. The $\mathrm{N}$-inputs must be equal to the ratio between the memory distortion duration and the sample time of the digital predistorter. A reduction of the memory size may be achieved by validation of the introduced model for memory non-linear amplifier and it will be object of further studies. However, RF techniques based on stabilisation of the amplifier operating point results in an effective way to counteract for the memory effects. The action of an RF compensation circuit on the DAB Class AB amplifier is shown in Fig.2(c). The memory distortions are almost cancelled and the residual gain compression (AM/AM) can be effectively counteracted by baseband 1dimensional predistortion.

\section{Measurements and Simulation Results}

Fig.3, 4, and 5 show the amplifier output spectrum for different OBO values with and without non-linearity counteractions. The ACI level is one of the transmitter quality parameters. ACI at $970 \mathrm{KHz}$ from the central frequency must be $30 \mathrm{~dB}$ below the carriers level. This constraint, however, does not represent an absolute limitation on the OBO choice for the predistorted amplifier. Indeed ACI at 970 $\mathrm{KHz}$ could be attenuated (even if hardly and expensively) by RF filtering. In-Band intermodulations, vice versa, cannot be filtered out and cause Bit Error Rate degradation at the receiver side. Consequently it is possible to select the OBO in order to reach optimum performance, ones the memory effects are eliminated and $\mathrm{AM} / \mathrm{AM}$ and $\mathrm{AM} / \mathrm{PM}$ are counteracted by the predistorter. It has been experienced that DAB systems need to reach an uncoded Bit Error Rate of $1.0 * 10^{-}$ 5 to provide a good quality audio. The optimum OBO could then be chosen in order to minimise the Total Degradation defined as

$$
T D=\left[\left(E_{b} / N_{o}\right)_{O B O}-\left(E_{b} / N_{o}\right)_{l i n}\right]+O B O
$$

where $\left(\mathrm{E}_{\mathrm{b}} / \mathrm{N}_{\mathrm{o}}\right)_{\text {lin }}$ and $\left(\mathrm{E}_{\mathrm{b}} / \mathrm{N}_{\mathrm{o}}\right)_{\text {OBO }}$ represents (respectively in linear and clipping 
predistorted environment) the signal to noise ratio needed to reach the $1.0 * 10^{-5}$ BER performance. TD versus $\mathrm{OBO}$ can be derived by use of Fig.6 and an optimum $\mathrm{OBO}$ of about $4 \mathrm{~dB}$ can be estimated. However, this $\mathrm{OBO}$ value gives no more than $25 \mathrm{~dB}$ of ACI (see Fig.4) and a severe RF filtering at the amplifier output could be needed. Moreover, it is important to remind that usually a total Implementation Loss lower than $1 \mathrm{~dB}$ is required for $\mathrm{DAB}$ transmitter. Therefore an $\mathrm{OBO}$ value higher than $5.5 \mathrm{~dB}$ appears to be a more reasonable choice. Indeed the Implementation Loss for this OBO due to non linear distortions is about 0.4 $\mathrm{dB}$, leaving $0.6 \mathrm{~dB}$ margin for further losses (i.e.: local oscillators phase noise,...).
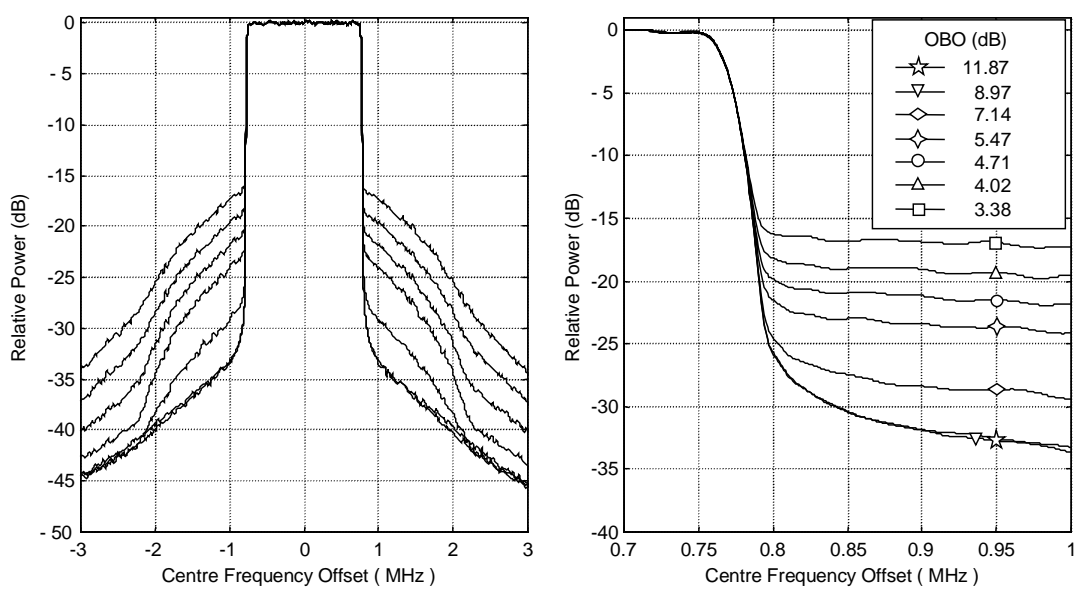

Fig. 3 DAB Spectrum without Predistortion (Simulation without Memory Effect)
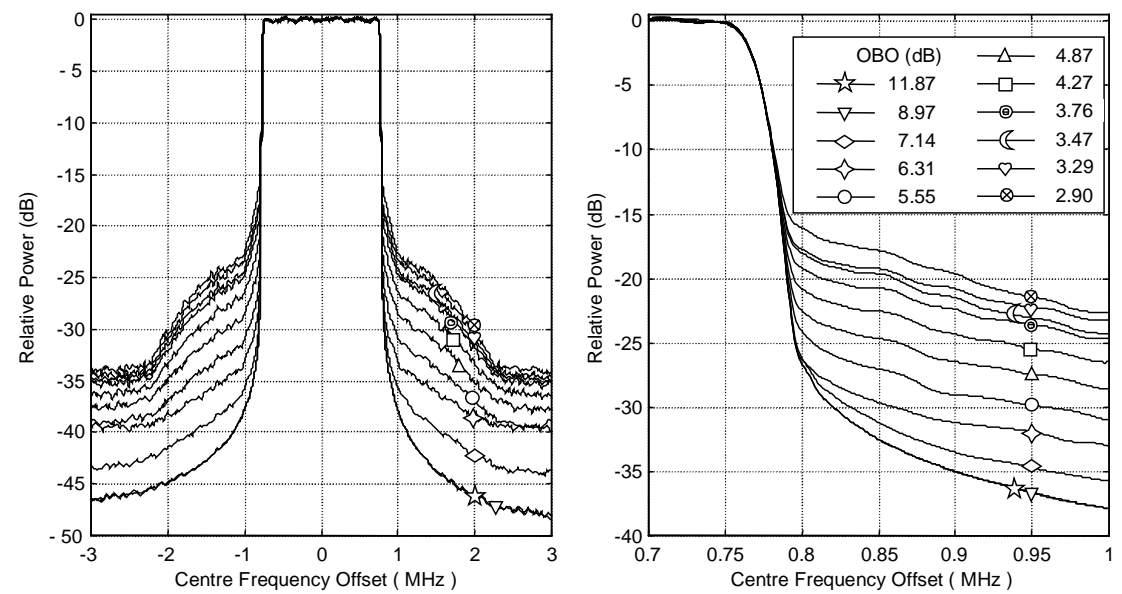

Fig. 4 DAB Spectrum with Predistortion (Simulation without Memory Effect) 

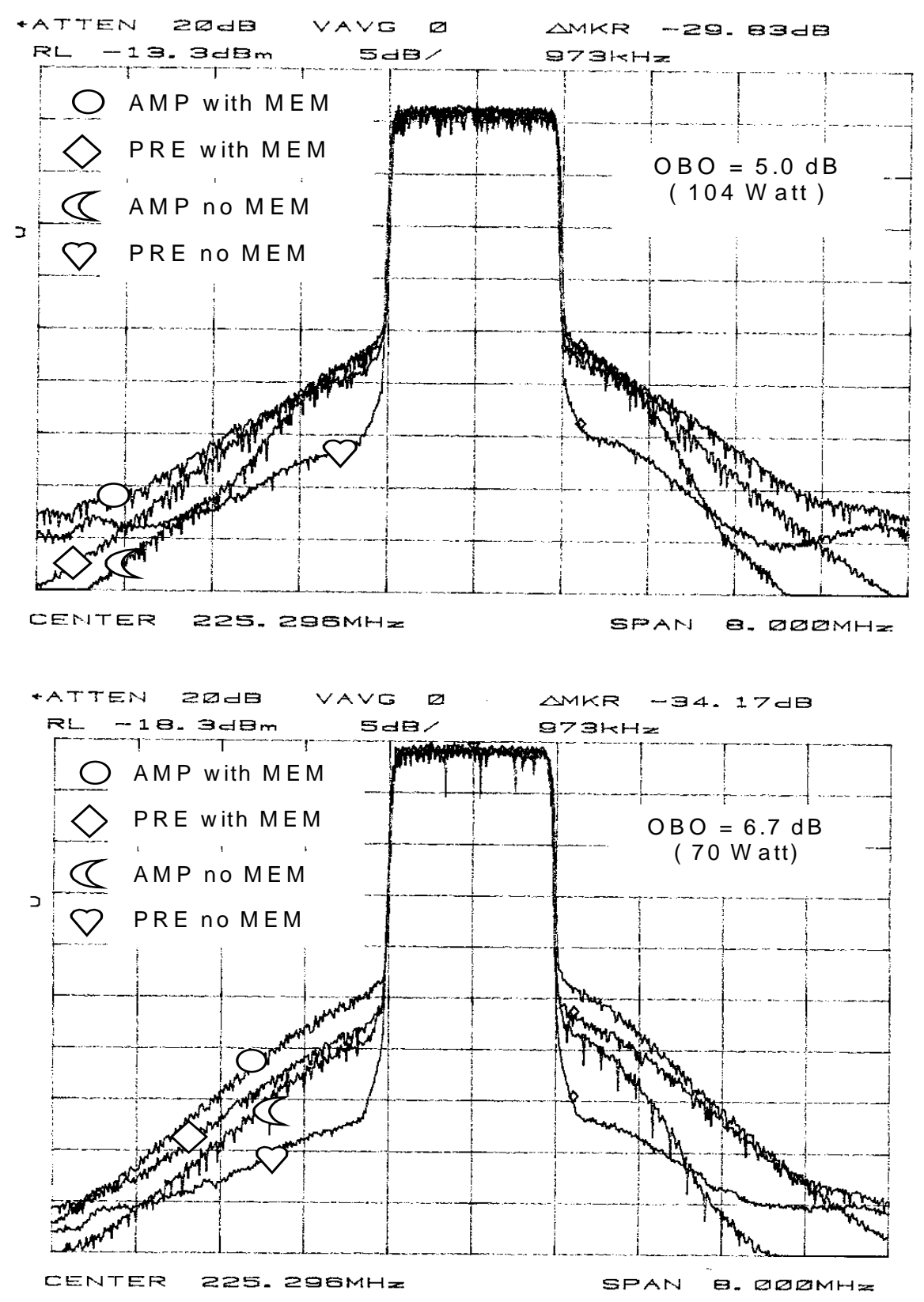

Fig. 5 Measured Output Spectrum: (a) $\mathrm{OBO}=5.0 \mathrm{~dB}$ (b) $\mathrm{OBO}=6.7 \mathrm{~dB}$ 

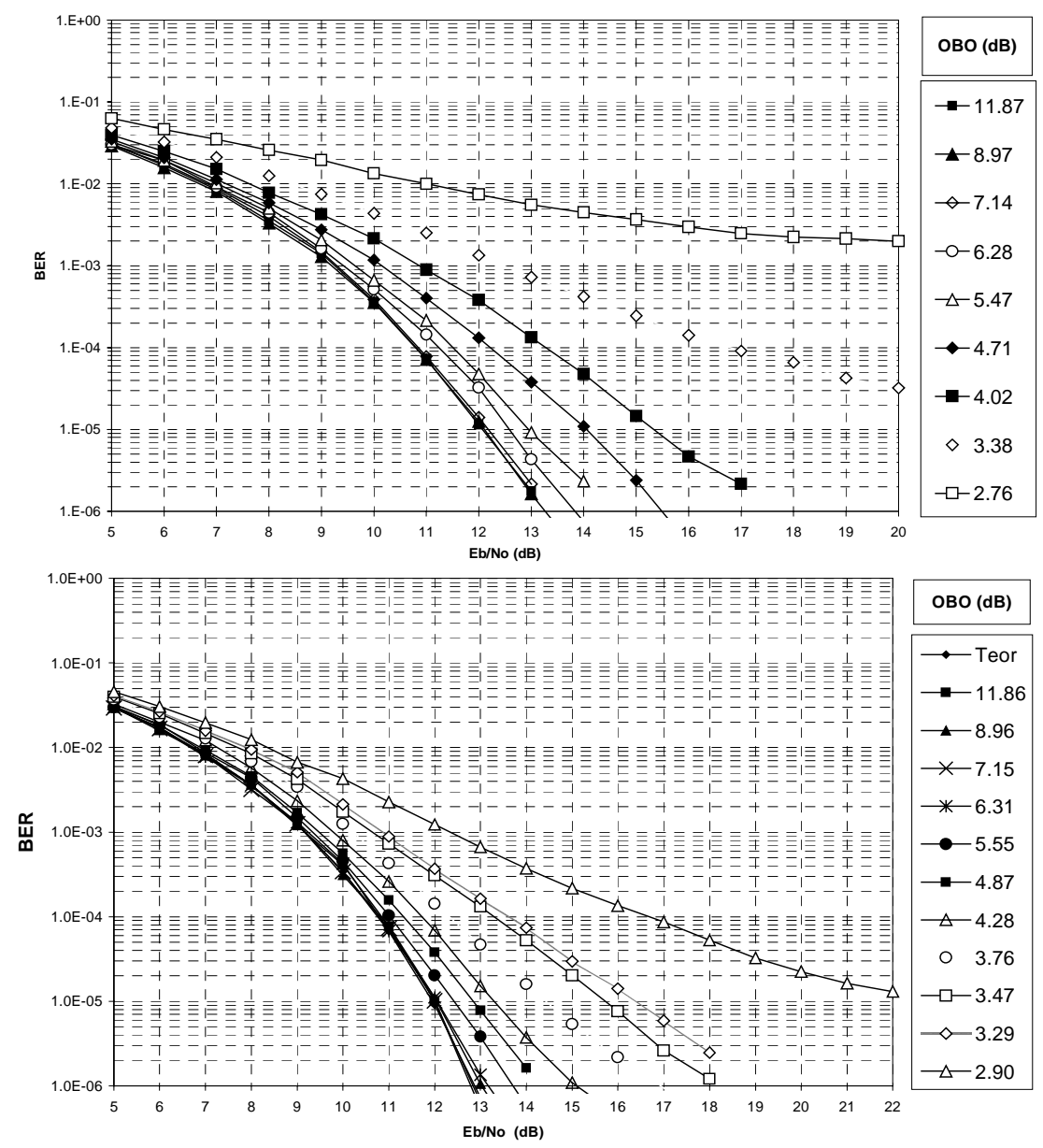

Fig. 6 DAB Performance in AWGN Channel without Memory Effect (a) No Predistortion (b) With Predistortion

\section{Conclusions}

The sensitivity of DAB-COFDM signal to non-linear distortions has been widely investigated. The effectiveness of baseband AM/AM and AM/PM predistortion combined with RF memory compensation has been outlined. Vice versa, the amplifier is not much sensitive to the predistorter action, if memory effects are not counteracted. Moreover, the good agreement between measured and simulated amplifier output spectrum testifies the quality of Itelco realisation of both digital baseband predistorter and RF memory compensation circuit. Finally, a way to choose the optimum OBO value has been suggested. The introduced idea for memory effects modelling and correspondent digital baseband counteraction will be object of further studies and it will be presented in future works. 


\section{References}

1. W Honcharenko et al., "Broadband Wireless Access", IEEE Communications Magazine, Vol. 35, No 1, Jenuary 1997 pp. 20-26

2. CRL, FUB et al., CABSINET ACTS Project, D21.1 State of the Art Report, Limited Deliverable

3. DVB, "Framing Structure, Channel Coding and Modulation for Digital Terrestrial Television", DVB Document A012, June 1996.

4. ETS 300 401, "Radio Broadcasting Systems; Digital Audio Broadcasting (DAB) to mobile portable and fixed receivers", February 1995, pp180.

5. A.R. Kaye, D.A. George, M.J. Eric, "Analysis and Compensation of Bandpass Nonlinearities for Communications", IEEE Trans. on Comm., VOL. COM-20, October 1972, pp. 965-972.

6. M. Schetzen, "The Volterra and Wiener theories of nonlinear systems", New York, Wiley \& Sons 1980

7. E. Biglieri, S. Barberis, M. Catena, "Analysis and compensation of nonlinearities in digital transmission systems", IEEE JSAC, Vol. SAC-8, n.1, Jan 1988, pp. 42-51.

8. A. Sarti, S. Pupolin, "Recursive Techniques for the Synthesis of a pth-order inverse of a Volterra System", ETT Vol. 3 n.4, Jul./Aug. 1992, pp. 315-322.

9. A.A.M.Saleh and J.Salz, "Adaptive Linearisation of Power Amplifiers in Digital Radio Systems", The Bell Syst. Tech. Journal, Vol. 62, No. 4, April 1983, pp. 1019-1033.

10. J.K.Cavers, "Amplifiers Linearization Using a Digital Preedistorter with Fast Adaptation and Low Memory Requirements", IEEE Trans. on Veh. Tech., Vol. VT-39, No. 4, November 1990, pp. 374-382.

11. G.Karam and H.Sari, "Generalised Data Predistortion Using Intersymbol Interpolation", Philips Res. Journal, Vol.46, 1991, pp.1-22.

12. M. Faulkner, M. Yohansson, "Adaptive Linearization Using Predistortion Experimental Results", IEEE Trans. on Veh. Tech., Vol. VT-43 n.2, May. 1994, pp. 323332.

13. S.Andreoli, P.Banelli, S.Cacopardi, H.G. McClure, "Digital Linearizer for RF Amplifiers", IEEE Trans. on Broad., Vol. 43, No 1 March 1997, pp 12-19.

14. J. Rinne, and M. Renfors, "The Behaviour of Orthogonal Frequency Division Multiplexing Signals in Amplitude Limiting Channel", IEEE International Conference on Communications (ICC '94), New Orleans, USA, pp. 381-385. 